\title{
X-RAY INSIGHTS INTO THE NATURE OF WEAK EMISSION-LINE QUASARS AT HIGH REDSHIFT
}

\author{
Ohad Shemmer ${ }^{1,6}$, W. N. Brandt ${ }^{1}$, Scott F. Anderson ${ }^{2}$, Aleksandar M. Diamond-Stanic ${ }^{3}$, Xiaohui Fan $^{3}$, \\ Gordon T. Richards ${ }^{4}$, Donald P. Schneider ${ }^{1}$, and Michael A. Strauss ${ }^{5}$ \\ ${ }^{1}$ Department of Astronomy \& Astrophysics, The Pennsylvania State University, University Park, PA 16802, USA \\ ${ }^{2}$ Department of Astronomy, University of Washington, Box 351580, Seattle, WA 98195, USA \\ ${ }^{3}$ Steward Observatory, University of Arizona, 933 North Cherry Avenue, Tucson, AZ 85721, USA \\ ${ }^{4}$ Department of Physics, Drexel University, 3141 Chestnut Street, Philadelphia, PA 19104, USA \\ 5 Princeton University Observatory, Peyton Hall, Princeton, NJ 08544, USA \\ Received 2008 September 3; accepted 2009 February 8; published 2009 April 16
}

\begin{abstract}
We present Chandra observations of nine high-redshift quasars $(z=2.7-5.9)$ discovered by the Sloan Digital Sky Survey with weak or undetectable high-ionization emission lines in their UV spectra (WLQs). Adding archival X-ray observations of six additional sources of this class has enabled us to place the strongest constraints yet on the X-ray properties of this remarkable class of active galactic nuclei (AGNs). Although our data cannot rule out the possibility that the emission lines are overwhelmed by a relativistically boosted continuum, as manifested by BL Lac objects, we find that WLQs are considerably weaker in the X-ray and radio bands than the majority of BL Lacs found at much lower redshifts. If WLQs are high-redshift BL Lacs, then it is difficult to explain the lack of a large parent population of X-ray and radio bright weak-lined sources at high redshift. We also consider the possibility that WLQs are quasars with extreme properties, and in particular that the emission lines are suppressed by high accretion rates. Using joint spectral fitting of the X-ray spectra of 11 WLQs, we find that the mean photon index in the hard X-ray band is consistent with those observed in typical radio-quiet AGNs with no hint of an unusually steep hard-X-ray spectrum. This result poses a challenge to the hypothesis that WLQs have extremely high accretion rates, and we discuss additional observations required to test this idea.
\end{abstract}

Key words: galaxies: active - galaxies: nuclei - quasars: emission lines - quasars: general - X-rays: galaxies

Online-only material: machine-readable table

\section{INTRODUCTION}

Since strong and broad emission lines are a hallmark of quasar optical-UV spectra, the discovery of $\sim 50$ Sloan Digital Sky Survey (SDSS; York et al. 2000) quasars at $z \sim 2.7-5.9$ with extremely weak or undetectable emission lines (hereafter WLQs) is puzzling (see Fan et al. 1999 for the discovery of the prototype high-redshift WLQ, SDSS J153259.96-003944.1, at $z=4.62$ ). While the rest-frame equivalent widths (EWs) of the Ly $\alpha+\mathrm{N}$ v emission lines of typical SDSS quasars at $z \sim 3$ follow a log-normal distribution and 68\% of EWs are in the range $\sim 40-100 \AA$ (Fan et al. 2001; Diamond-Stanic et al. 2009), WLQs exhibit $\mathrm{EW}(\operatorname{Ly} \alpha+\mathrm{N} v)<10 \AA$, constituting a $4 \sigma$ deviation from the mean of the distribution (there is no detectable corresponding deviation at the high EW end; Diamond-Stanic et al. 2009). By virtue of their largely featureless spectra, the redshifts of these WLQs can be determined only from the onset of the Ly $\alpha$ forest or the Lyman limit (i.e., $z>2.2$ for SDSS sources; e.g., Fan et al. 1999; Anderson et al. 2001; Collinge et al. 2005; Schneider et al. 2005).

Since WLQs exhibit typical quasar UV continua with no signs of broad C IV absorption, they are unlikely to be dustobscured or broad-absorption line quasars (see, e.g., Anderson et al. 2001; Collinge et al. 2005). In addition, Shemmer et al. (2006; hereafter S06) have argued against the possibility that these sources are high-redshift galaxies with apparent quasarlike luminosities due to gravitational-lensing amplification, or lensed quasars with continua amplified by microlensing. S06 suggested that the two most likely interpretations for

\footnotetext{
6 Current address: Department of Physics, University of North Texas, Denton, TX 76203, USA; ohad@unt.edu.
}

the weakness of the high-ionization emission lines in these sources are either line dilution due to a relativistically boosted continuum or, alternatively, physical suppression of the highionization emission lines.

The first interpretation for the nature of WLQs involves relativistic beaming of the continuum emission from a jet directed close to our line of sight, as observed in BL Lacertae objects (BL Lacs). This scenario implies that the SDSS may have discovered the long-sought, high-redshift BL Lacs (e.g., Stocke 2001); the absence of such sources is even more puzzling since their beamed continua should have made them easier to detect at high redshift than ordinary quasars. However, this interpretation faces serious difficulties. For example, S06 have found that the basic X-ray, optical, and radio properties of five WLQs are consistent only with the X-ray- and radio-weak tail of the BL Lac population. Moreover, while both WLQs and BL Lacs share the property of a lineless UV spectrum, BL Lacs also often exhibit high optical polarization levels, rapid and large-amplitude flux variations, and spectral energy distributions (SEDs) dominated by beamed, non-thermal emission at all wavelengths (e.g., Urry \& Padovani 1995). Such extreme properties have, so far, not been observed in a subsample of SDSS WLQs (e.g., Fan et al. 1999; Diamond-Stanic et al. 2009).

The alternative interpretation is that WLQs may be quasars with abnormal photoionizing continuum or broad emission-line region (BELR) properties. In this scenario, the weak highionization emission lines of WLQs may, for example, ultimately be a consequence of exceptionally high accretion rates in these sources. A higher accretion rate is expected to result in a softer, UV-peaked continuum that suppresses the highionization emission lines, while low-ionization species such as 


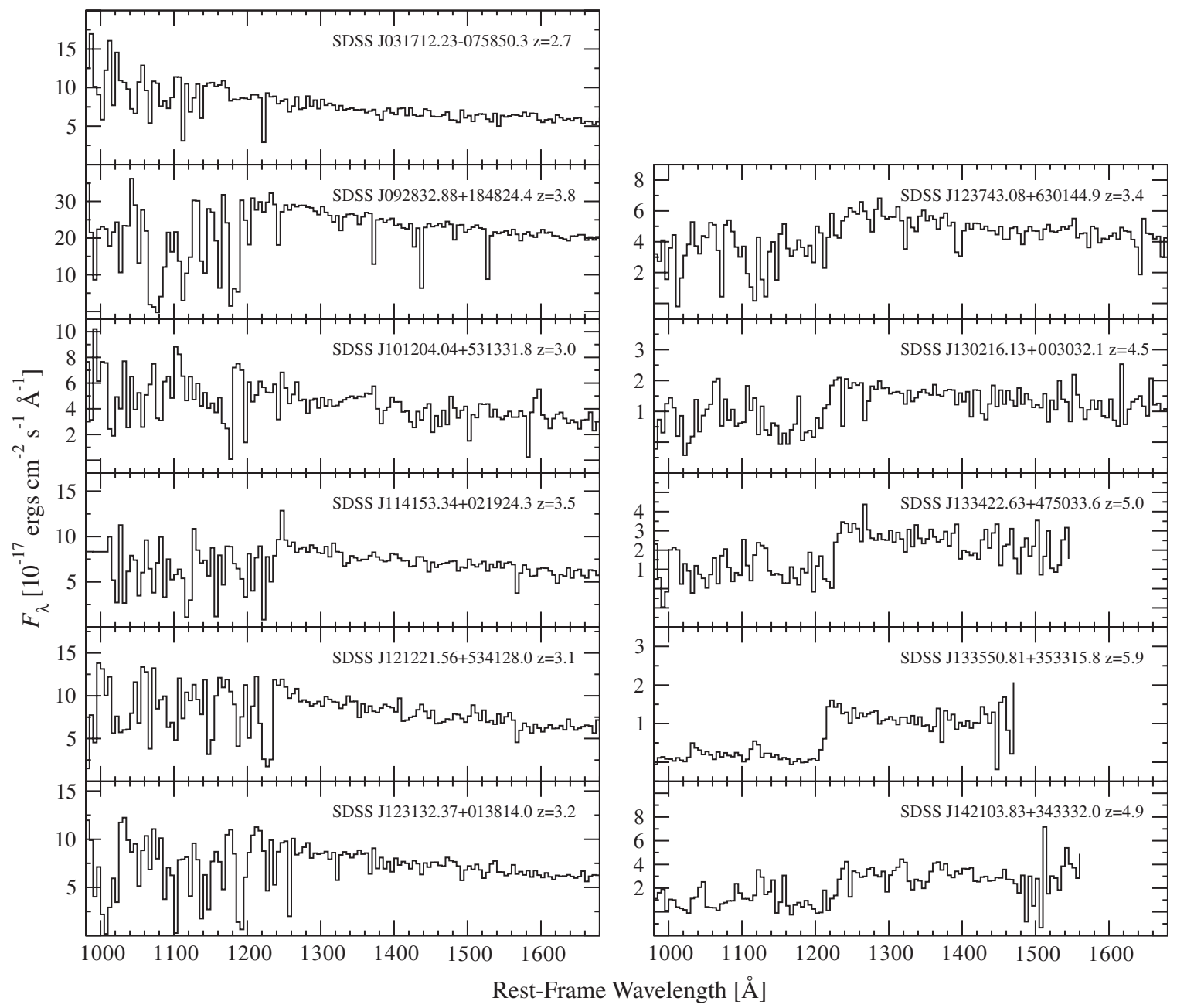

Figure 1. Ultraviolet spectra of the WLQ sample resampled in bins of $5 \AA$, for clarity. Spectra were obtained from the SDSS archive, except for SDSS J1335+3533, for which the spectrum was obtained from the Keck observatory (Fan et al. 2006).

the Balmer and $\mathrm{Fe}$ II lines are not significantly affected (e.g., Leighly et al. 2007a, 2007b). Accretion rates in WLQs have not yet been determined in order to test this possibility (for example, by measuring the width of $\mathrm{H} \beta$ in such sources; see, e.g., Shemmer et al. 2004).

The key to understanding WLQs, therefore, lies in comparing their multiwavelength spectral, as well as polarimetric and temporal, properties with those of BL Lacs and typical quasars. While the SDSS WLQs are rare, their exceptional properties constitute a challenge to our overall understanding of active galactic nucleus (AGN) physics and thus might lead to new insights about the accretion process, emission-line formation, and relativistic jets. In this paper, we measure basic X-ray properties for the first respectably sized sample of WLQs in order to shed light on their nature and to assist in planning more detailed X-ray spectroscopy of these sources in the future. Since $\mathrm{X}$-rays probe the innermost regions of AGNs, our observations are intended to study the basic diagnostics of the central accretion process in WLQs. This paper nearly triples the number of $z>2.2$ WLQs that have sensitive X-ray coverage, increasing the number of X-ray detections by almost a factor of 5 .

This paper is organized as follows: in Section 2 we describe the WLQ sample selection, as well as our X-ray observations and their reduction; our basic findings are presented in Section 3. In Section 4 we discuss the main results emerging from our
X-ray study of WLQs and the clues they provide on the nature of these sources; a summary is given in Section 5. Complete WLQ names are given in the tables and figures, and their abbreviated versions are used throughout the text. Throughout this work we compute luminosity distances using the standard cosmological model with parameters $H_{0}=70 \mathrm{~km} \mathrm{~s}^{-1} \mathrm{Mpc}^{-1}, \Omega_{\Lambda}=0.7$, and $\Omega_{M}=0.3$.

\section{SAMPLE SELECTION, OBSERVATIONS, AND DATA REDUCTION}

\subsection{Targeted Chandra Observations}

We selected nine WLQs at $z=2.7-5.9$ for short $(\sim 4-23$ ks) Chandra observations in Cycle 8 (2007-2008); the restframe ultraviolet spectra of these sources appear in Figure 1. The Chandra observation log appears in Table 1, where we also list the papers in which the sources were first identified as WLQs or BL Lac candidates. These sources were selected from the SDSS archive for being the brightest sources of this class; except for SDSS J1302+0030, these were not targeted in $\mathrm{X}$-rays prior to Cycle 8. SDSS J1302+0030 was not detected in a 10.81 ks Chandra Cycle 4 exposure; our additional 10.50 ks exposure in Cycle 8 has enabled its detection (see below). SDSS J1335+3533 was discovered by Fan et al. (2006) and is the only source in this sample that was not detected with the SDSS 
Table 1

Chandra Observation Log

\begin{tabular}{|c|c|c|c|c|c|c|}
\hline $\begin{array}{l}\text { Object } \\
(\text { SDSS J) } \\
\end{array}$ & $z$ & $\begin{array}{l}\Delta_{\text {Opt-X }}{ }^{a} \\
(\operatorname{arcsec})\end{array}$ & $\begin{array}{c}\text { X-ray } \\
\text { Obs. Date }\end{array}$ & $\begin{array}{l}\text { Chandra } \\
\text { Obs. ID }\end{array}$ & $\begin{array}{c}\text { Exp. Time }{ }^{b} \\
(\mathrm{ks})\end{array}$ & Ref \\
\hline $031712.23-075850.3$ & 2.7 & 0.1 & 2007 Mar 17 & 7780 & 4.42 & 1 \\
\hline $092832.90+184824.4$ & 3.8 & 0.1 & 2007 Jan 2 & 779 & .98 & 2 \\
\hline 21924.3 & 3.5 & 0.2 & 2007 Feb 10 & 7777 & 3.99 & 1 \\
\hline $121221.56+534128.0$ & 3.1 & 0.4 & $2007 \mathrm{Jul} 24$ & 7778 & 4.13 & 1 \\
\hline $123132.38+013814.0$ & 3.2 & 0.1 & 2007 Jul 5 & 7781 & 4.09 & 1 \\
\hline $130216.13+003032.1^{\mathrm{c}}$ & 4.5 & $0.6^{\mathrm{d}}$ & 2007 Feb 24 & 7784 & 10.50 & 3 \\
\hline $133422.63+475033.6$ & 5.0 & 0.1 & 2007 Aug 25 & 7885 & 11.60 & 3 \\
\hline $133550.81+353315.8$ & 5.9 & 0.2 & 2008 Mar 10 & 7783 & 23.47 & 4 \\
\hline $142103.83+343332.0$ & 4.9 & 0.3 & 2008 Feb 27 & 7782 & 12.79 & 5 \\
\hline
\end{tabular}

Notes. The optical positions of the quasars have been obtained from the reference given in the seventh column, and the X-ray positions have been obtained with WAVDETECT.

a Angular distance between the optical and X-ray positions.

b The Chandra exposure time has been corrected for detector dead time.

${ }^{c}$ A 10.81 ks Chandra Cycle 4 exposure of the source (Obs. ID 3958) is not reflected in this Table. The Cycle 8 observation raises the total exposure time on the source to $21.31 \mathrm{ks}$, and the merged event file is used in subsequent analyses throughout the paper (see S06 and Section 2 for details).

${ }^{\mathrm{d}}$ Based on a merged image composed of Chandra Cycle 4 and Cycle 8 exposures.

References. (1) Collinge et al. 2005; (2) this work; (3) Schneider et al. 2005; (4) Fan et al. 2006; (5) Schneider et al. 2007.

quasar-selection algorithm (Richards et al. 2002; this algorithm is not designed to detect quasars above $z \simeq 5.4$ ).

The nine WLQs were observed with the Advanced CCD Imaging Spectrometer (ACIS; Garmire et al. 2003) with the S3 CCD at the aimpoint. Faint mode was used for the event telemetry format in all the observations, and ASCA grade 0,2, 3,4 , and 6 events were used in the analysis, which was carried out using standard $\mathrm{CIAO}^{7}$ V3.4 routines. No background flares are present in these observations. Source detection was carried out with WAVDETECT (Freeman et al. 2002) using wavelet transforms (with wavelet scale sizes of $1,1.4,2,2.8$, and 4 pixels) and a false-positive probability threshold of $10^{-4}$. Given the small number of pixels being searched due to the accurate a priori source positions and the subarcsecond on-axis angular resolution of Chandra, the probability of spurious detections is extremely low; most of the sources were in fact detected at a false-positive probability threshold of $10^{-6}$. The $\mathrm{X}$-ray positions of the detected sources differ by $00^{\prime} 1-00^{\prime \prime} 6$ from their optical positions (Table 1). These results are consistent with expected Chandra positional errors.

The X-ray counts detected in the ultrasoft band (0.3-0.5 $\mathrm{keV})$, soft band $(0.5-2 \mathrm{keV})$, hard band $(2-8 \mathrm{keV})$, and full band $(0.5-8 \mathrm{keV})$ are reported in Table 2 . The counts were derived from manual aperture photometry, and they are consistent with the photometry obtained using WAVDETECT. Except for SDSS J1302+0030, all of the targeted WLQs were detected with 3-110 full-band counts. A significant detection of SDSS J1302+0030 (using WAVDETECT with a false-positive probability threshold of $10^{-4}$ ) was achieved only by merging the Cycle 8 exposure (with three full-band counts) with that from Cycle 4 (with two full-band counts; see S06); the $\mathrm{X}$-ray counts for this source, presented in Table 2, were obtained from the merged image. Table 2 also includes the band ratio, which is the ratio between the counts in the hard band

\footnotetext{
7 Chandra Interactive Analysis of Observations. See http://cxc.harvard.edu/ciao/
}

and the counts in the soft band, and the effective power-law photon index $\left(\Gamma\right.$, where $N(E) \propto E^{-\Gamma} ; \Gamma$ was calculated from the band ratio using the Chandra PIMMs v3.9d tool ${ }^{8}$ ) for each source.

Finally, we searched for rapid variability within the Chandra observations of all WLQs detected with > 10 full-band counts by applying a Kolmogorov-Smirnov (KS) test to the photon arrival times against the null hypothesis of a constant rate, but no significant flux variations were detected. This result is not unexpected given the combination of the relatively short observations (15-30 minutes in the rest-frame) and the small number of detected photons from most of the sources.

\subsection{Serendipitous XMM - Newton Observations}

Two additional SDSS WLQs at $z>2.2$ were serendipitously observed by XMM-Newton (Jansen et al. 2001). SDSS $\mathrm{J} 1012+5313$ at $z=3.0$ is a newly identified WLQ from SDSS Data Release 5 (Schneider et al. 2007). The source was observed with XMM-Newton on 2001 April 19 for 18.7 ks (dataset ID 0111100201; PI M. Watson). SDSS J1237+6301 at $z=3.4$ was identified as a BL Lac candidate by Collinge et al. (2005). The source was observed with XMM-Newton on 2006 October 25 for $20.7 \mathrm{ks}$ (dataset ID 0402250101; PI B. Maughan). The XMM-Newton observations were processed using standard XMM-Newton Science Analysis System ${ }^{9}$ v7.1.0 tasks. The SDSS J1012+5313 observation included only MOS1 and MOS2 images, and the entire observation was carried out during a period of flaring activity; hence no time filtering was employed. The SDSS J1237+6301 observation was filtered in time to remove periods of background flaring in which the count rates of each MOS (pn) detector exceeded 0.35 (1.0) counts $\mathrm{s}^{-1}$ for events having $E>10 \mathrm{keV}$; the net exposure times were $10.7 \mathrm{ks}$ and $4.9 \mathrm{ks}$ for the MOS1/MOS2 and pn detectors, respectively. We detected SDSS J1012+5313 at $7^{\prime} .0$ from the XMM-Newton aimpoint in both the MOS1 and MOS2 detectors. For each detector, source counts were extracted from an aperture with a $30^{\prime \prime}$ radius centered on the source, and background counts were extracted from an aperture with a similar size in a nearby sourcefree region. SDSS J1237+6301 was not detected in any of the $X M M$-Newton detectors. The upper limit on the X-ray flux of the source was taken as the square root of the number of counts encircled within an aperture of $30^{\prime \prime}$ centered on the optical position of the source (which is offset by 9.8 from the aimpoint) multiplied by three (i.e., a $3 \sigma$ upper limit). The X-ray counts of SDSS J1012+5313 as well as the upper limit on the number of counts of SDSS J1237+6301 were corrected for vignetting using the exposure maps due to the large offsets from the aimpoints. The SDSS spectra of the two sources appear in Figure 1.

\section{RESULTS}

\subsection{Basic Multiwavelength Properties of the WLQ Sample}

Table 3 lists the basic X-ray, optical, and radio properties of our WLQ sample:

Column (1). The SDSS J2000.0 quasar coordinates, accurate to $\sim 0$ '.1 (Pier et al. 2003).

Column (2). The Galactic column density in units of $10^{20} \mathrm{~cm}^{-2}$, taken from Dickey \& Lockman (1990) and obtained with the HEASARC $N_{\mathrm{H}}$ tool. $^{10}$

\footnotetext{
8 See http://cxc.harvard.edu/toolkit/pimms.jsp.

$9 \mathrm{http}: / / \mathrm{xmm}$.esac.esa.int/sas.

$10 \mathrm{http}: / /$ heasarc.gsfc.nasa.gov/cgi-bin/Tools/w3nh/w3nh.pl.
} 
Table 2

X-ray Counts, Band Ratios, and Effective Photon Indices

\begin{tabular}{|c|c|c|c|c|c|c|}
\hline \multirow{2}{*}{$\begin{array}{l}\text { Object } \\
\text { (SDSS J) }\end{array}$} & \multicolumn{4}{|c|}{ X-ray Counts ${ }^{\mathrm{a}}$} & \multirow[b]{2}{*}{ Band Ratio $^{\mathrm{b}}$} & \multirow[b]{2}{*}{$\Gamma^{\mathrm{b}}$} \\
\hline & $0.3-0.5 \mathrm{keV}$ & $0.5-2 \mathrm{keV}$ & $2-8 \mathrm{keV}$ & $0.5-8 \mathrm{keV}$ & & \\
\hline $092832.90+184824.4$ & $<3.0$ & $52.2_{-7.2}^{+8.3}$ & $10.9_{-3.2}^{+4.6}$ & $63.1_{-7.9}^{+9.0}$ & $0.21_{-0.07}^{+0.09}$ & $2.2_{-0.3}^{+0.6}$ \\
\hline $121221.56+534128.0$ & $<3.0$ & $3.0_{-1.6}^{+2.99^{3}}$ & $<3.0$ & $3.0_{-1.6}^{+2.94}$ & $<1.00$ & $>0.7$ \\
\hline $123132.38+013814.0$ & $10.9_{-3.2}^{+4.4}$ & $83.6_{-9.1}^{+10.2}$ & $26.5_{-5.1}^{+6.2}$ & $109.9_{-10.4}^{+11.5}$ & $0.32_{-0.07}^{+0.08}$ & $1.7_{-0.2}^{+0.2}$ \\
\hline $130216.13+003032.1^{\mathrm{c}}$ & $<3.0$ & $2.0_{-13}^{+2.7}$ & $<8.0$ & $5.0_{-2.2}^{+3.4}$ & $<4.00$ & $>-0.6$ \\
\hline $142103.83+343332.0$ & $<3.0$ & $2.0_{-1.3}^{+2.7}$ & $<4.8$ & $3.8_{-1.9}^{+3.1}$ & $<2.40$ & $>-0.1$ \\
\hline
\end{tabular}

Notes.

${ }^{a}$ Errors on the X-ray counts were computed according to Tables 1 and 2 of Gehrels (1986) and correspond to the $1 \sigma$ level; these were calculated using Poisson statistics. The upper limits are at the 95\% confidence level and were computed according to Kraft et al. (1991). Upper limits of 3.0, 4.8, 6.4, and 8.0 indicate that $0,1,2$, and $3 \mathrm{X}$-ray counts, respectively, have been found within an extraction region of radius 1 " centered on the optical position of the quasar (considering the background within this source-extraction region to be negligible).

${ }^{\mathrm{b}}$ We calculated errors at the $1 \sigma$ level for the band ratio (the ratio between the $2-8 \mathrm{keV}$ and $0.5-2 \mathrm{keV}$ counts) and effective photon index following the "numerical method" described in Section 1.7.3 of Lyons (1991); this avoids the failure of the standard approximate-variance formula when the number of counts is small (see Section 2.4.5 of Eadie et al. 1971). The photon indices have been obtained applying the correction required to account for the ACIS quantum-efficiency decay at low energy.

${ }^{c}$ The X-ray counts, band ratio, and photon index for this source were obtained from a merged event file of our new Chandra Cycle 8 observation and an archival Chandra Cycle 4 observation (see Section 2 for more details). The total exposure time of the merged event file is $21.31 \mathrm{ks}$.

Column (3). The monochromatic $A B$ magnitude at a rest-frame wavelength of $1450 \AA\left(A B_{1450}=-2.5 \log f_{1450 \AA}-48.6\right.$; Oke $\&$ Gunn 1983). All the magnitudes have been corrected for Galactic extinction (Schlegel et al. 1998). Except for SDSS $\mathrm{J} 1335+3533$, for which this magnitude has been obtained from a Keck spectrum, all the magnitudes were obtained from SDSS Data Release 5 spectra and a fiber light-loss correction has been applied; this was calculated as the difference between the synthetic $i$ magnitude (i.e., the integrated flux across the $i$ bandpass in the SDSS spectrum) and the photometric SDSS $i$ magnitude, assuming no flux variation between the photometric and spectroscopic epochs.

Column (4). The absolute $B$-band magnitude, computed assuming a UV-optical ( 1200-4000 A) power-law slope of $\alpha_{\mathrm{UV}}=-0.5\left(f_{v} \propto v^{\alpha_{\mathrm{Uv}}}\right.$; Vanden Berk et al. 2001).

Column (5) and (6). The flux density and monochromatic luminosity at a rest-frame wavelength of $2500 \AA$, computed from the magnitudes in Column 3, assuming a UV-optical power-law slope as in Column 4.

Column (7) and (8). The count rate in the observed-frame 0.5-2 keV band and the corresponding flux, corrected for Galactic absorption. The fluxes have been calculated using PIMMs, assuming a power-law model with $\Gamma=2.0$.

Column (9) and (10). The flux density and monochromatic luminosity at a rest-frame energy of $2 \mathrm{keV}$, computed assuming $\Gamma=2.0$ in the entire Chandra band $(0.5-8 \mathrm{keV})$.

Column (11). The luminosity in the rest-frame $2-10 \mathrm{keV}$ band. Column (12). The optical-to-X-ray power-law slope, $\alpha_{\mathrm{ox}}$, defined as

$$
\alpha_{\mathrm{ox}}=\frac{\log \left(f_{2 \mathrm{keV}} / f_{2500 \AA}\right)}{\log \left(\nu_{2 \mathrm{keV}} / \nu_{2500 \AA}\right)}
$$

where $f_{2 \mathrm{keV}}$ and $f_{2500 \AA}$ are the flux densities at rest-frame 2 $\mathrm{keV}$ and $2500 \AA$, respectively.

Column (13). The difference between the measured $\alpha_{\text {ox }}$ (from Column 12) and the predicted $\alpha_{\mathrm{ox}}$, given the UV luminosity from Column 6 , based on the observed $\alpha_{\text {ox }}-L_{v}(2500 \AA)$ relation in AGNs (given as Equation (3) of Just et al. 2007; see also Steffen et al. 2006). The statistical significance of this difference is also given in units of $\sigma$, where $\sigma=$ 0.146 for $31<\log L_{v}(2500 \AA)<32$, and $\sigma=0.131$ for $32<\log L_{v}(2500 \AA$ ) $<33$ (see Table 5 of Steffen et al. 2006; these differences do not account for flux-density measurement errors).

Column (14). The radio-to-optical power-law slope, $\alpha_{\text {ro }}$, defined as

$$
\alpha_{\mathrm{ro}}=\frac{\log \left(f_{5 \mathrm{GHz}} / f_{2500 \AA}\right)}{\log \left(\nu_{5 \mathrm{GHz}} / \nu_{2500 \AA}\right)} .
$$

This parameter can be directly converted to the radio-loudness parameter $R$ (where $R=f_{5 \mathrm{GHz}} / f_{4400 \AA}$; Kellermann et al. 1989) in the following way:

$$
\alpha_{\mathrm{ro}}=\frac{\log \left[R\left(\frac{2500}{4400}\right)^{\alpha_{\mathrm{UV}}}\right]}{\log \left(\nu_{5 \mathrm{GHz}} / \nu_{2500 \AA}\right)}
$$

where we take $\alpha_{\mathrm{UV}}=-0.5$ (as in Column 4). The flux density at a rest-frame frequency of $5 \mathrm{GHz}$ was computed from the FIRST (Becker et al. 1995) flux density at an observed-frame frequency of $1.4 \mathrm{GHz}$, if available. Otherwise, the NVSS (Condon et al. 1998) flux density at the same observed band was used. We assume a radio power-law slope of $\alpha_{\mathrm{r}}=-0.5\left(f_{v} \propto v^{\alpha_{\mathrm{r}}}\right.$; e.g., Rector et al. 2000). Upper limits on $\alpha_{\text {ro }}$ are at the $3 \sigma$ level, since the positions of all our sources are known a priori. The flux densities at rest frame $4400 \AA$ were computed from the $A B_{1450}$ magnitudes assuming $\alpha_{\mathrm{UV}}=-0.5$ (as in Column 4). Typical $\alpha_{\text {ro }}$ values are $<-0.39$ (i.e., $R>100$ ) for radio-loud quasars (RLQs) and $>-0.21$ (i.e., $R<10$ ) for radio-quiet quasars (RQQs). Seven of our sources are RQQs; four sources are radio intermediate with $-0.39 \lesssim \alpha_{\text {ro }} \lesssim-0.21$ (i.e., $10 \lesssim R \lesssim 100$ ).

\subsection{The X-ray Spectrum of SDSS J1231+0138}

SDSS J1231+0138 is the only WLQ in our sample with sufficient X-ray counts to allow an investigation of its $\mathrm{X}$-ray spectrum. The spectrum of the source was extracted with 
Table 3

X-ray, Optical, and Radio Properties

\begin{tabular}{|c|c|c|c|c|c|c|c|c|c|c|c|c|c|}
\hline $\begin{array}{l}\text { Object (SDSS J) } \\
\text { (1) }\end{array}$ & $\begin{array}{l}N_{\mathrm{H}^{\mathrm{a}}} \\
(2)\end{array}$ & $\begin{array}{l}A B_{1450} \\
\text { (3) }\end{array}$ & $\begin{array}{c}M_{B} \\
(4)\end{array}$ & $\begin{array}{c}f_{2500 \AA^{\mathrm{b}}} \\
(5)\end{array}$ & $\begin{array}{c}\log \left(v L_{v}\right) \\
2500 \AA \\
(6)\end{array}$ & $\begin{array}{l}\text { Count } \\
\text { Rate }^{c} \\
(7)\end{array}$ & $\begin{array}{l}f_{\mathrm{x}}^{\mathrm{d}} \\
(8)\end{array}$ & $\begin{array}{l}f_{2 \mathrm{keV}^{\mathrm{e}}} \\
(9)\end{array}$ & $\begin{array}{c}\log \left(v L_{v}\right) \\
2 \mathrm{keV} \\
(10)\end{array}$ & $\begin{array}{c}\log L \\
2-10 \mathrm{keV} \\
(11)\end{array}$ & $\begin{array}{l}\alpha_{\mathrm{OX}} \\
(12)\end{array}$ & $\begin{array}{c}\Delta \alpha_{\text {ox }}(\sigma)^{\mathrm{f}} \\
\quad(13)\end{array}$ & $\begin{array}{l}\alpha_{\mathrm{ro}}{ }^{\mathrm{g}} \\
(14)\end{array}$ \\
\hline $031712.23-075850.3$ & 5.14 & 18.7 & -27.1 & 15.8 & 46.5 & $5.40_{-1.10}^{+1.35}$ & $22.1_{-4.5}^{+5.5}$ & 12.19 & 45.0 & 45.2 & -1.58 & $+0.12(0.8)$ & $>-0.23^{1}$ \\
\hline+184824.4 & 3.83 & 17.4 & -28.9 & 52.1 & 47.2 & & & 37.03 & 45.7 & 45.9 & -1.59 & $+0.21(1.6)$ & -0.24 \\
\hline $101204.04+531331.8^{\mathrm{h}}$ & 0.78 & 19.3 & -26.6 & 9.2 & 46.3 & $3.28_{-1.86}^{+4.85 \mathrm{k}}$ & $20.8_{-11.8}^{+30.7}$ & 12.37 & 45.1 & 45.3 & -1.49 & $+0.18(1.3)$ & -0.28 \\
\hline $114153.34+021924.3$ & 2.30 & 18.5 & -27.6 & 18.4 & 46.7 & $7.18_{-1.33}^{+1.66}$ & $\begin{array}{l}27.1_{-5.1}^{+6.1} 8 \\
0\end{array}$ & 18.07 & 45.3 & 45.5 & -1 & $+0.19(1.3)$ & -0.24 \\
\hline $121221.56+534128.0$ & 1.71 & 18.6 & -27.3 & 17.2 & 46.6 & $0.73_{-0.39}^{+0.71}$ & & 1.65 & 44.2 & 44.4 & -1.93 & $-0.21(1.5)$ & $>-0.08$ \\
\hline $123132.38+013814.0$ & 1.81 & 18.6 & -27.4 & 17.5 & 46.7 & $20.44_{-2.23}^{+2.49}$ & $76.0_{-8.3}^{+9.3}$ & 47.94 & 45.7 & 45.9 & -1.37 & $+0.35(2.4)$ & -0.32 \\
\hline $123743.08+630144.9^{\mathrm{h}}$ & 1.66 & 19.0 & -27.1 & 12.0 & 46.5 & $<3.30^{\mathrm{k}}$ & $<16.6$ & $<10.88$ & $<45.1$ & $<45.3$ & $<-1.55$ & $<+0.15(<1.0)$ & $>-0.12$ \\
\hline $130216.13+003032.1^{\mathrm{i}}$ & 1.57 & 19.5 & -27.0 & 7.3 & 46.5 & $0.09_{-0.06}^{+0.12}$ & $0.3_{-0.2}^{+0.5}$ & 0.28 & 43.7 & 43.9 & -2.08 & $-0.38(2.6)$ & $>-0.16$ \\
\hline $133422.63+475033.6$ & 1.63 & 19.1 & -27.6 & 10.7 & 46.7 & $19_{-0.32}^{+0.41}$ & & 3.90 & 44.9 & 45.1 & -1.70 & $+0.02(0.2)$ & $>-0.13$ \\
\hline $133550.81+353315.8$ & 0.92 & $19.8^{\mathrm{j}}$ & -27.3 & 5.8 & 46.6 & & & 0.48 & 44.1 & 44.3 & -1.95 & $-0.24(1.7)$ & $>-0.18$ \\
\hline $142103.83+343332.0$ & 1.16 & 19.0 & -27.8 & 12.5 & 46.8 & $0.16_{-0.10}^{+0.21}$ & $0.6_{-0.4}^{-0.3}$ & 0.50 & 44.0 & 44.2 & -2.07 & $-0.33(2.3)$ & $>-0.11$ \\
\hline
\end{tabular}

Notes.

${ }^{a}$ Neutral Galactic absorption column density in units of $10^{20} \mathrm{~cm}^{-2}$ taken from Dickey \& Lockman (1990).

${ }^{b}$ Flux density at rest-frame $2500 \AA$ in units of $10^{-28} \mathrm{erg} \mathrm{cm}^{-2} \mathrm{~s}^{-1} \mathrm{~Hz}^{-1}$.

${ }^{c}$ Observed count rate computed in the $0.5-2 \mathrm{keV}$ band in units of $10^{-3}$ counts s$^{-1}$

${ }^{\mathrm{d}}$ Galactic absorption-corrected flux in the observed $0.5-2 \mathrm{keV}$ band in units of $10^{-15} \mathrm{erg} \mathrm{cm}^{-2} \mathrm{~s}^{-1}$.

${ }^{\mathrm{e}}$ Flux density at rest-frame $2 \mathrm{keV}$ in units of $10^{-32} \mathrm{erg} \mathrm{cm}^{-2} \mathrm{~s}^{-1} \mathrm{~Hz}^{-1}$.

${ }^{\mathrm{f}}$ The difference between measured and predicted $\alpha_{\mathrm{ox}}\left(\Delta \alpha_{\mathrm{ox}}\right)$, and the significance of that difference $(\sigma)$, based on the Just et al. (2007) $\alpha_{\mathrm{ox}}-L_{v}(2500 \AA)$ relation.

${ }^{g}$ Unless otherwise noted, radio-to-optical flux ratios $\left(\alpha_{\mathrm{ro}}\right.$; see Section 3.1 for the conversion of these values to the radio-loudness parameter $R$ ) involve radio flux densities at an observed-frame frequency of $1.4 \mathrm{GHz}$ taken from the FIRST survey (Becker et al. 1995); upper limits on $\alpha_{\text {ro }}$ are calculated from the $3 \sigma$ FIRST detection threshold at the source position.

${ }^{\mathrm{h}} \mathrm{X}$-ray data obtained from serendipitous XMM-Newton observations.

${ }^{i}$ The X-ray count rate, fluxes, luminosities, and $\alpha_{\mathrm{ox}}$ were obtained from the merged event file of two Chandra exposures (see Section 2).

j Obtained from the Keck spectrum of Fan et al. (2006).

${ }^{\mathrm{k}}$ Count rate corrected for the exposure map.

${ }^{1}$ Flux density at an observed-frame frequency of $1.4 \mathrm{GHz}$ taken from the NVSS survey (Condon et al. 1998); upper limits are calculated from the NVSS detection threshold of $2.5 \mathrm{mJy}$.

the CIAO task PSEXTRACT using a circular region of 2" radius centered on the X-ray centroid. The background was extracted from a source-free annulus with inner and outer radii of $5^{\prime \prime}$ and $25^{\prime \prime}$, respectively. The spectrum was binned in groups of 10 counts per bin. We used XSPEC v11.3.2 (Arnaud 1996) to fit the spectrum with a power-law model and a Galactic-absorption component, which was kept fixed during the fit $\left(N_{\mathrm{H}}=1.81 \times 10^{20} \mathrm{~cm}^{-2}\right.$; Dickey \& Lockman 1990). The spectrum and its best-fit model and residuals appear in Figure 2. The best-fit photon index is $\Gamma=1.76_{-0.34}^{+0.35}$ with $\chi^{2}=5.57$ for 8 degrees of freedom; this photon index is consistent with the value obtained from the band ratio (Table 2) and is typical for radio-intermediate AGNs such as SDSS J1231+0138 $\left(\alpha_{\text {ro }}=-0.32\right.$; see Section 3.1; e.g., Reeves \& Turner 2000). Adding an intrinsic-absorption component at the redshift of the source did not improve the fit; the upper limit on the intrinsic absorption is $N_{\mathrm{H}} \lesssim 5.70 \times 10^{22} \mathrm{~cm}^{-2}$ (at $90 \%$ confidence).

\subsection{Mean X-ray Spectral Properties of High-Redshift WLQs}

Since all of our WLQs, except for SDSS J1231+0138 (see Section 3.2), lack sufficient X-ray counts for individual spectral analyses, we have obtained mean X-ray spectral constraints for high-redshift WLQs as a group by jointly fitting their X-ray spectra (e.g., Vignali et al. 2005; S06). Eleven sources with Chandra spectra were included in the joint-fitting process, including the nine WLQs targeted in this work (i.e., all sources from Tables 1 and 2 including the merged dataset of SDSS J1302+0030) and the only other two Chandra-detected WLQs from S06, namely SDSS J140850.91+020522.7 and SDSS J144231.72+011055.2 (see Tables 1-3 of S06). The combined

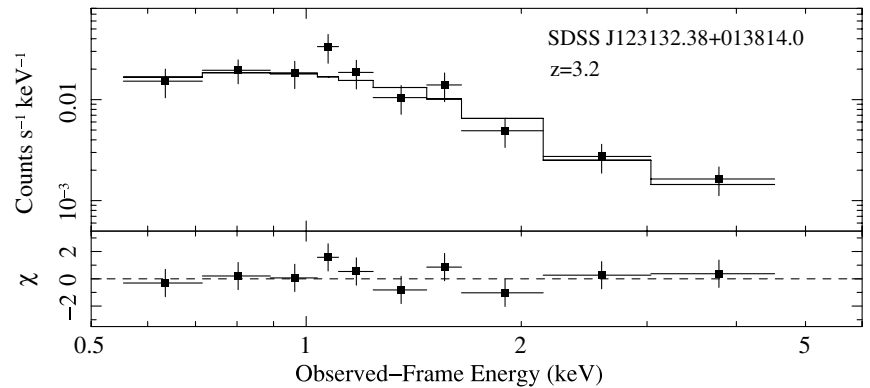

Figure 2. Chandra spectrum of SDSS J1231+0138. Filled squares represent the binned spectrum, and the solid histogram represents the best-fit Galacticabsorbed power-law model. The $\chi$ residuals are in units of $\sigma$ with error bars of size 1.

spectrum consists of a total of 350 full-band counts, obtained in $107 \mathrm{ks}$. We note that there are only two other X-ray-detected WLQs with XMM-Newton spectra: SDSS J1012+5313 (see Section 2.2) and SDSS J004054.65-091526.8 (S06); these spectra do not provide a significant contribution to the spectral constraints since the first was taken during a period of flaring activity (Section 2.2) and the second was detected at low signalto-noise (with only $31.8 \pm 11.6$ net source counts; Schneider et al. 2003).

The X-ray spectra of all 11 WLQs were extracted as in Section 3.2 with PSEXTRACT using circular regions of $2^{\prime \prime}$ radius centered on the X-ray centroid of each source, and background counts were extracted using annuli of different sizes (to avoid contamination from nearby X-ray sources) centered on each source. We used XSPEC to fit the set of 11 unbinned spectra 
jointly with the following models: (1) a power-law model and a Galactic-absorption component (Dickey \& Lockman 1990; using the PHABS absorption model in XSPEC), which was kept fixed during the fit, and (2) a model similar to the first with an added intrinsic (redshifted) neutral-absorption component (using the ZPHABS model in XSPEC). We used the $C$-statistic (Cash 1979) in all the fits. The joint-fitting process associated with each quasar its own Galactic-absorption column density and redshift, and allowed the flux normalizations to vary freely while maintaining a fixed value of the power-law photon index. The errors associated with the best-fit X-ray spectral parameters are quoted at the $90 \%$ confidence level for one parameter of interest $(\Delta C=2.71$; Avni 1976; Cash 1979).

The joint-fitting process was carried out four times for both models. In the first run, we included the entire observed-energy range of all the spectra $(0.5-8 \mathrm{keV})$. In the second run, we included only the common rest-frame energy range of all 11 sources: $3.45 \lesssim E_{\text {rest }} \lesssim 29.60 \mathrm{keV}$ (this range is based upon the entire $0.5-8 \mathrm{keV}$ observed-frame band for each source, and is a consequence of the wide range of redshifts among the sources; see also S06). In the third run, we fitted the entire observedenergy range of ten WLQs, excluding SDSS J1231+0138 (since this source contributes $\sim 30 \%$ of the total counts and hence might bias the results). In the fourth run, we have restricted the fitting only to radio-quiet WLQs (i.e., excluding SDSS J0928+1848, SDSS J1141+0219, SDSS J1231+0138, and SDSS $\mathrm{J} 144231.72+011055.2)$ to minimize potential contribution from jet-related emission.

Table 4 lists the best-fit parameters from the joint-fitting process, and a contour plot of the $\Gamma-N_{\mathrm{H}}$ parameter space for the first run is shown in Figure 3. Based on $F$-tests, we find that the inclusion of an intrinsic-absorption component (in addition to a Galactic absorbed power-law model) does not significantly improve any of the fits; hence no significant intrinsic absorption is detected in any of the joint fits. Table 4 shows the mean photon index of WLQs is not significantly different from typical values for RQQs or radio-intermediate AGNs (e.g., Reeves \& Turner 2000; Page et al. 2005; Vignali et al. 2005). In particular, there is no hint of an exceptionally high photon index as might have been expected if WLQs are high accretion-rate sources (see Section 4.2). The hard-X-ray spectra of AGN can appear flattened if Compton reflection is not taken into account in the spectral fitting. Since the fractional contribution from Compton reflection generally weakens with increasing luminosity, we do not expect that the typical value we obtain for the mean photon index of our luminous, high-redshift WLQs is strongly affected

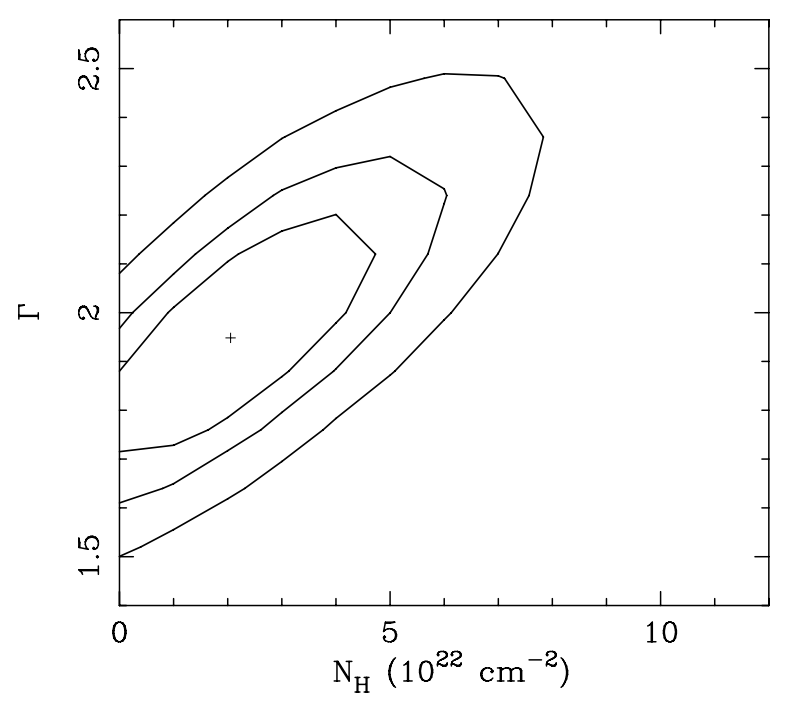

Figure 3. $68 \%, 90 \%$, and $99 \%$ confidence regions for the photon index vs. intrinsic column density derived from joint spectral fitting of our sample of 11 WLQs over their entire observed energy range.

by Compton reflection (e.g., Iwasawa \& Taniguchi 1993; Zhou \& Wang 2005; Bianchi et al. 2007; Shemmer et al. 2008).

The exclusion of SDSS J1231+0138 or the exclusion of all radio-intermediate sources have no significant effect on the results (see also Section 3.2). In addition, photon indices derived in individual runs are consistent with each other, within the errors; these errors are large due to the limited number of sources in our sample and the short exposures. We conclude that the mean X-ray spectral properties of high-redshift WLQs are consistent with those observed in typical, radio- quiet-tointermediate AGNs.

\section{DISCUSSION}

Our primary goal is to use X-ray information to shed light on the nature of WLQs at high redshift. The Chandra and serendipitous XMM-Newton observations have provided significantly stronger constraints on the X-ray luminosities of our WLQs than previous ROSAT observations (see also Collinge et al. 2005; S06). This should allow us to scrutinize the classification of the WLQs and determine the most probable explanation for the absence of their high-ionization lines. The two most likely scenarios for the absence of the UV lines are the association of WLQs with BL Lac objects or the hypothesis that

Table 4

Best-Fit Parameters from Joint Fitting of High-Redshift WLQs

\begin{tabular}{|c|c|c|c|c|}
\hline XSPEC Model & Sources Included & $\begin{array}{l}\text { Intrinsic } N_{\mathrm{H}} \\
\left(10^{22} \mathrm{~cm}^{-2}\right)\end{array}$ & $\Gamma$ & $C$-statistic (bins) \\
\hline PHABS+POW & all (11 sources) & $\ldots$ & $1.79_{-0.16}^{+0.17}$ & $191.4(294)$ \\
\hline PHABS+POW, common energy range ${ }^{a}$ & all (11 sources) & $\ldots$ & $1.96_{-0.22}^{+0.22}$ & $170.4(260)$ \\
\hline PHABS + POW & all but SDSS J1231+0138 & $\cdots$ & $1.79_{-0.22}^{+0.22}$ & $133.0(214)$ \\
\hline PHABS+POW & seven radio-quiet WLQs ${ }^{\mathrm{b}}$ & $\ldots$ & $1.81_{-0.43}^{+0.45}$ & $48.2(84)$ \\
\hline PHABS+ZPHABS+POW & all (11 sources) & $\leqslant 5.08$ & $1.95_{-0.28}^{+0.49}$ & $189.9(294)$ \\
\hline PHABS+ZPHABS+POW, common energy range ${ }^{\mathrm{a}}$ & all (11 sources) & $\leqslant 4.34$ & $1.96_{-0.22}^{+0.26}$ & $170.4(260)$ \\
\hline PHABS+ZPHABS+POW & all but SDSS J1231+0138 & $\leqslant 7.05$ & $1.97_{-0.35}^{+0.37}$ & $131.8(214)$ \\
\hline PHABS+ZPHABS+POW & seven radio-quiet WLQs ${ }^{b}$ & $\leqslant 7.74$ & $1.86_{-0.48}^{+0.33}$ & $48.2(84)$ \\
\hline
\end{tabular}

Notes.

a The fitting was performed only for the common rest-frame energy range among all sources (3.45-29.60 keV).

b Excluding the radio-intermediate WLQs SDSS J0928+1848, SDSS J1141+0219, SDSS J1231+0138, and SDSS J144231.72+011055.2. 
WLQs are quasars with extreme properties (see Section 1; S06). Given the X-ray, optical, and radio luminosities presented in this work for an extended sample of 15 high-redshift WLQs (i.e., including 11 sources from this work and four sources from S06), we are now in a position to perform meaningful comparisons of these basic properties with those of BL Lacs.

\subsection{Comparing WLQs with BL Lacs}

For the purpose of comparing our WLQs to BL Lac objects, we have compiled a dataset of X-ray, optical, and radio luminosities for a sample of 279 BL Lacs from a variety of surveys, covering most of the current BL Lac selection methods (see, e.g., Perlman et al. 2001). We selected only BL Lacs with reliable redshifts from the Einstein Slew survey (11 sources; e.g., Perlman et al. 1996), the Einstein Medium Sensitivity Survey (EMSS; 24 sources; e.g., Rector et al. 2000), the ROSAT All-Sky Survey-Green Bank (RGB) catalog (47 sources; e.g., LaurentMuehleisen et al. 1999), the Deep X-ray Radio Blazar Survey (DXRBS; 16 sources; Perlman et al. 1998; Landt et al. 2001), and the SDSS (181 sources; Collinge et al. 2005; Plotkin et al. 2008). Most BL Lacs with reliable redshifts from the 1 Jy survey (e.g., Stickel et al. 1991), i.e., radio-selected BL Lacs, overlap with sources detected in the above surveys and are included in our sample. Basic X-ray, optical, and radio properties of our BL Lac sample are given in Table 5.

All fluxes and $K$-corrections for our BL Lac sample were computed assuming the spectral slopes $\alpha_{\mathrm{x}}=-1, \alpha_{\mathrm{o}}=-0.5$, and $\alpha_{\mathrm{r}}=-0.5$ (where $f_{v} \propto v^{\alpha}$ ) for the X-ray, optical$\mathrm{UV}$, and radio bands, respectively; the same as those assumed for our WLQs. These spectral slopes are consistent with the observed values in the majority of quasars, although in the optical and radio bands they are somewhat different from the slopes observed in typical BL Lacs (e.g., Sambruna et al. 1996; Rector et al. 2000; Reeves \& Turner 2000; Vanden Berk et al. 2001; Collinge et al. 2005). We repeated the optical and radio flux and $K$-correction calculations for the BL Lacs, this time assuming typical observed BL Lac slopes (i.e., $\alpha_{\mathrm{r}}=0.27$; e.g., Stickel et al. 1991; $\alpha_{\mathrm{o}}=-1.5$; e.g., Collinge et al. 2005), and found that the differences in optical and radio fluxes between the two sets of calculations were small (since the majority of these BL Lacs lie at $z \lesssim 0.3$ ). In any case, the differences in optical and radio fluxes between the calculations using different slopes are well within the uncertainties stemming from measurement errors, BL Lac variability, and beaming (note that, for consistent comparisons with the observed properties of the WLQs, we make no attempt to correct the fluxes or luminosities of the $\mathrm{BL}$ Lacs for the effects of relativistic beaming).

In Figure 4 we present X-ray, optical, and radio monochromatic luminosities versus redshift for our BL Lac comparison sample and our extended sample of 15 WLQs. In spite of the large difference in redshift between the majority of BL Lacs $(z \lesssim 0.3)$ and WLQs $(z \gtrsim 2.2)$, the WLQs do not have luminosities that are so high that they are disjoint from those of the BL Lacs; a few BL Lacs even reach the optical luminosities of WLQs. The fact that no BL Lacs have been found in the redshift range of our WLQs is not yet understood (e.g., Stocke \& Perrenod 1981; Stocke 2001; Padovani et al. 2007).

Are WLQs simply the long-sought high-redshift BL Lacs? In this scenario, it is difficult to explain why there are essentially no X-ray- and/or radio-bright WLQs in Figure 5, where we have plotted $\alpha_{\text {ox }}$ and $\alpha_{\text {ro }}$ for the BL Lacs and our WLQs in a 'color-color' diagram (the definitions of $\alpha_{\mathrm{ox}}$ and $\alpha_{\mathrm{ro}}$ in Section 3.1 are identical to the definitions used in S06, and note that the similar Figure 8 of S06 included many flat-spectrum radio sources and sources with unreliable redshifts). In other words, if the SDSS high-redshift WLQs are indeed the high-redshift tail of the BL Lac population with $\alpha_{\mathrm{ox}}$ and $\alpha_{\mathrm{ro}}$ distributions similar to their low-redshift counterparts, then a much larger, 'parent' population of X-ray- and radio-bright lineless sources at high redshift is still missing. Unless these are too optically faint (see below), the SDSS, for example, should have naturally discovered many such sources had they existed (see, e.g., Collinge et al. 2005; Anderson et al. 2007; Plotkin et al. 2008). Alternatively, if most BL Lacs at low redshift were X-ray and radio weak, and thus were largely missed by $\mathrm{X}$-ray and radio surveys, the SDSS should have discovered many members of this population as well (e.g., Collinge et al. 2005).

To test whether X-ray- and radio-bright BL Lacs are simply too faint and thus miss detection at high redshift, we looked for correlations between luminosity and $\alpha_{\mathrm{ox}}$ and $\alpha_{\mathrm{ro}}$ among our BL Lac sample. We found that $\alpha_{\mathrm{ox}}$ and $\alpha_{\text {ro }}$ are not significantly correlated with optical luminosity. On the other hand, $\alpha_{\mathrm{ox}}\left(\alpha_{\mathrm{ro}}\right)$ is significantly correlated with X-ray (radio) luminosity in the sense that, sources that are more $\mathrm{X}$-ray (radio) luminous are also X-ray (radio) brighter relative to their optical luminosities. These results suggest that we are not missing a putative, large parent population of BL Lacs at high redshift purely due to a

Table 5

X-ray, Optical, and Radio Properties of the BL Lac Sample

\begin{tabular}{|c|c|c|c|c|c|c|c|c|c|}
\hline BL Lac & $\begin{array}{c}\text { RA } \\
(\mathrm{J} 2000)\end{array}$ & $\begin{array}{c}\text { DEC } \\
(\mathrm{J} 2000)\end{array}$ & $z$ & $\begin{array}{c}\log \left[v L_{v}(2 \mathrm{keV})\right] \\
\left(\operatorname{erg~s}^{-1}\right)\end{array}$ & $\begin{array}{c}\log \left[v L_{v}(5500 \AA)\right] \\
\left(\operatorname{erg~s}^{-1}\right)\end{array}$ & $\begin{array}{c}\log \left[v L_{v}(5 \mathrm{GHz})\right] \\
\left(\operatorname{erg~s}^{-1}\right)\end{array}$ & $\alpha_{\mathrm{ox}}$ & $\alpha_{\text {ro }}$ & Reference \\
\hline SDSS J000157.23-103117.3 & 0.48850 & -10.52148 & 0.252 & $\leqslant 43.30$ & 44.05 & 41.53 & $\leqslant-1.35$ & -0.50 & 1 \\
\hline RGB J0007+472 & 2.00000 & 47.20222 & 0.280 & 43.95 & 44.33 & 41.81 & -1.21 & -0.50 & 2 \\
\hline SDSS J001736.91+145101.9 & 4.40378 & 14.85053 & 0.303 & $\leqslant 44.09$ & 44.70 & 42.03 & $\leqslant-1.30$ & -0.47 & 3 \\
\hline SDSS J002200.95+000657.9 & 5.50396 & 0.11610 & 0.306 & 44.24 & 44.03 & 40.42 & -0.99 & -0.30 & 1 \\
\hline WGA J0032.5-2849 & 8.13792 & -28.82222 & 0.324 & 43.68 & 44.06 & 42.34 & -1.21 & -0.65 & 4 \\
\hline WGA J0043.3-2638 & 10.84417 & -26.65139 & 0.451 & 44.12 & 45.02 & 42.40 & -1.41 & -0.48 & 4 \\
\hline SDSS J005620.07-093629.7 & 14.08366 & -9.60826 & 0.103 & 43.80 & 43.90 & 41.22 & -1.10 & -0.47 & 1 \\
\hline WGA J0100.1-3337 & 15.03917 & -33.62556 & 0.875 & 43.97 & 44.59 & 43.24 & -1.30 & -0.72 & 4 \\
\hline SDSS J010326.01+152624.8 & 15.85839 & 15.44021 & 0.246 & $\leqslant 43.81$ & 44.43 & 42.42 & $\leqslant-1.30$ & -0.59 & 3 \\
\hline RGB J0110+418 & 17.52000 & 41.83083 & 0.096 & 43.23 & 43.47 & 40.28 & -1.16 & -0.38 & 2 \\
\hline
\end{tabular}

Notes. Luminosities, $\alpha_{\mathrm{ox}}$, and $\alpha_{\text {ro }}$ were computed from the data given in the references (last column) using the $K$-corrections outlined in Section 4.1 . References. (1) Plotkin et al. 2008; (2) Laurent-Muehleisen et al. 1999; (3) Collinge et al. 2005; (4) Perlman et al. 1998.

(This table is available in its entirety in a machine-readable form in the online journal. A portion is shown here for guidance regarding its form and content.) 
No. 1, 2009

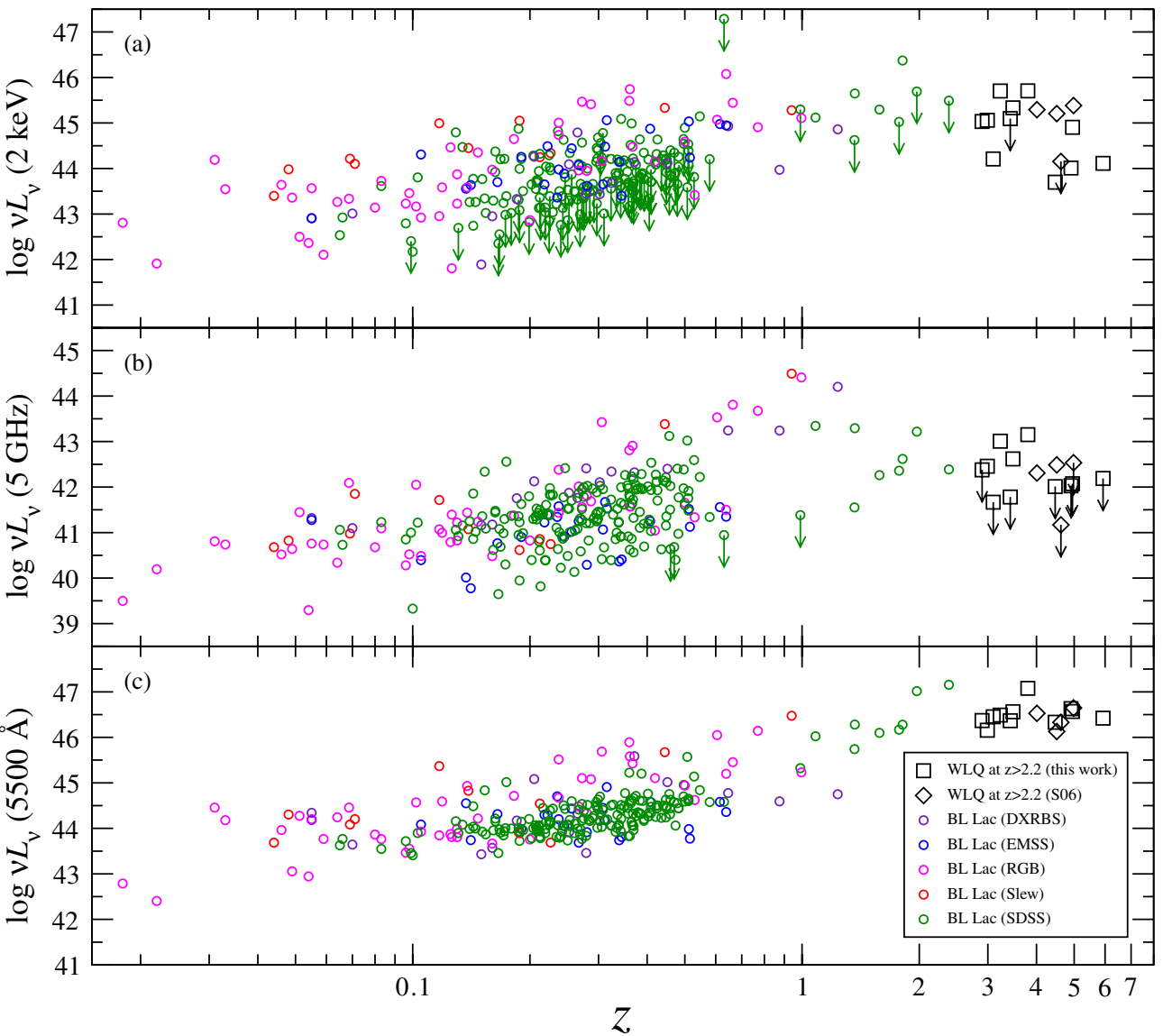

Figure 4. Monochromatic luminosities (in units of $\operatorname{erg~s}^{-1}$ ) in the X-ray (a), radio (b), and optical (c) bands versus redshift for BL Lacs and WLQs. Squares mark $z>2.2$ WLQs from this work, and diamonds mark $z>2.2$ WLQs from S06. Violet, blue, magenta, and red circles mark BL Lacs from the DXRBS (Perlman et al. 1998; Landt et al. 2001), EMSS (e.g., Rector et al. 2000), RGB (e.g., Laurent-Muehleisen et al. 1999), and the Einstein Slew (e.g., Perlman et al. 1996) surveys, respectively. Green circles mark higher confidence SDSS BL Lac candidates with reliable redshifts from the SDSS (Collinge et al. 2005; Plotkin et al. 2008). Upper limits are marked with down-pointing arrows.

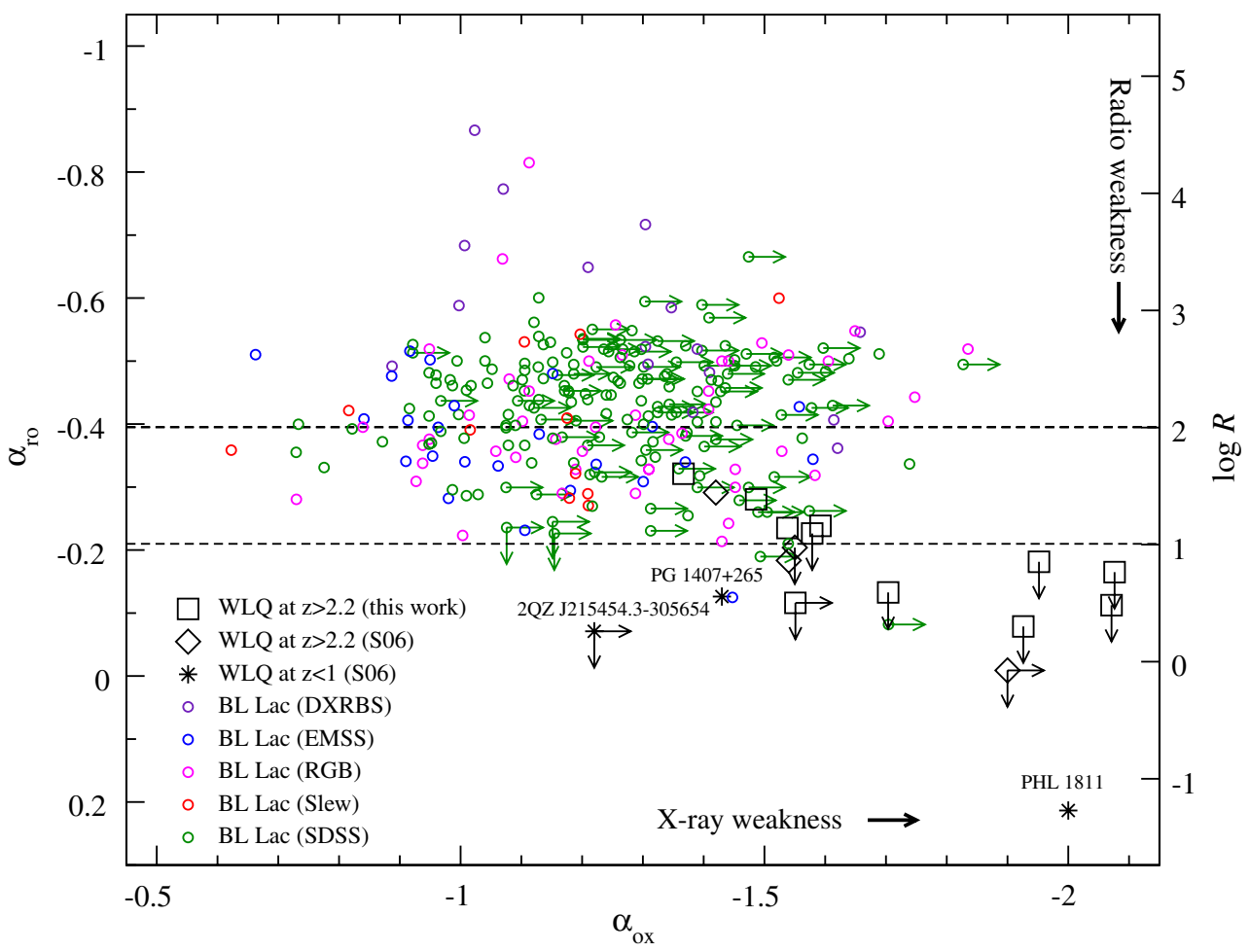

Figure 5. $\alpha_{\mathrm{ox}}-\alpha_{\mathrm{ro}}$ diagram for BL Lacs and WLQs. Symbols are similar to those in Figure 4. WLQs at $z<1$ from S06 are marked with asterisks. Right (down) pointing arrows mark $\alpha_{\mathrm{ox}}\left(\alpha_{\mathrm{ro}}\right)$ upper limits. All sources that lie above (below) the upper (lower) dashed line are radio loud (quiet) with $R>100(R<10)$. 


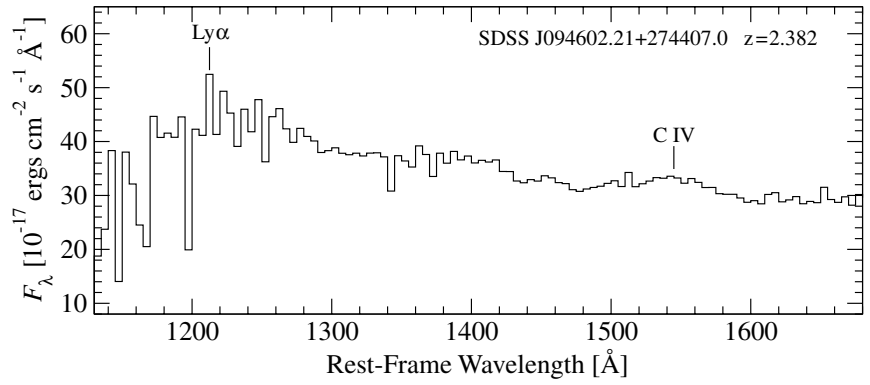

Figure 6. SDSS spectrum of the BL Lac candidate SDSS J094602.21+274407.0 from Plotkin et al. (2008), which may also be classified as a WLQ. The spectrum has been resampled in bins of $5 \AA$ (as in Figure 1). The locations of two prominent UV emission lines have been marked.

luminosity effect. An additional difficulty in associating WLQs with BL Lacs arises from the fact that, while there appears to be a trend of a positive X-ray-radio dependence among WLQs, there is no corresponding trend between the $\mathrm{X}$-ray and radio emission of BL Lacs (i.e., for a given $\alpha_{\mathrm{ox}}$ value, a BL Lac can have a wide range of $\alpha_{\text {ro }}$ values and vice versa; Figure 5; see also Plotkin et al. 2008).

Are WLQs related to the rare population of radio-weak BL Lacs (see, e.g., Collinge et al. 2005; Anderson et al. 2007)? Four BL Lacs from our comparison sample, SDSS J095125.90+ 504559.7 at $z=1.363$, SDSS J094602.21+274407.0 at $z=2.382$, SDSS J145326.52 +545322.4 at $z=0.100$ (Plotkin et al. 2008), and MS 2306.1-2236 at $z=0.137$ (Rector et al. 2000) have $\alpha_{\text {ro }}>-0.2$ and satisfy the radio-weak BL Lac (as well as radio-quiet AGN) classification; see also Table 5 of Collinge et al. (2005) for additional possible candidates from this group. Based on its redshift and UV properties, SDSS J094602.21+274407.0 may be classified as a high-redshift WLQ (it is the radio-weakest source with an $\alpha_{\text {ox }}$ upper limit from the SDSS BL Lac sample in Figure 5); we plot its SDSS spectrum in Figure 6. Further testing the association of WLQs with radio-weak BL Lacs requires broader spectroscopic coverage of both classes. For example, near-IR spectra of WLQs should be acquired to search for low-ionization emission lines in the rest-frame UV-optical band, and UV spectra of low-redshift, radio-weak BL Lacs should be obtained to measure the strengths of high-ionization emission lines. However, such observations will not resolve the missing parent population of 'typical' (i.e., $\mathrm{X}$-ray- and radio-bright) BL Lacs at high redshift, which remains an outstanding problem for any model attempting to unify WLQs and BL Lacs.

\subsection{Are WLQs Extreme Quasars?}

The second scenario attempting to explain the missing UV lines in WLQs suggests that these sources are quasars hosting abnormal BELRs or quasars with extreme properties (Section 1). In this case, one does not expect to find many radio-bright (i.e., radio-loud) WLQs, since the majority of quasars at all luminosities and redshifts are known to be radio quiet (e.g., Jiang et al. 2007). Hence, unless emission-line strength was correlated with radio properties, there is no problem of a missing parent population (see Section 4.1). In addition, the WLQs in our sample show blue UV continua with an average slope $\alpha_{\mathrm{UV}}=-0.8$, consistent with the slopes observed for high-redshift quasars (e.g., Schneider et al. 2001) as well as with the slopes of a larger sample of high-redshift WLQs (DiamondStanic et al. 2009). This apparently steeper slope (compared with $\alpha_{\mathrm{UV}}=-0.5$ observed for low-redshift quasars) is essentially

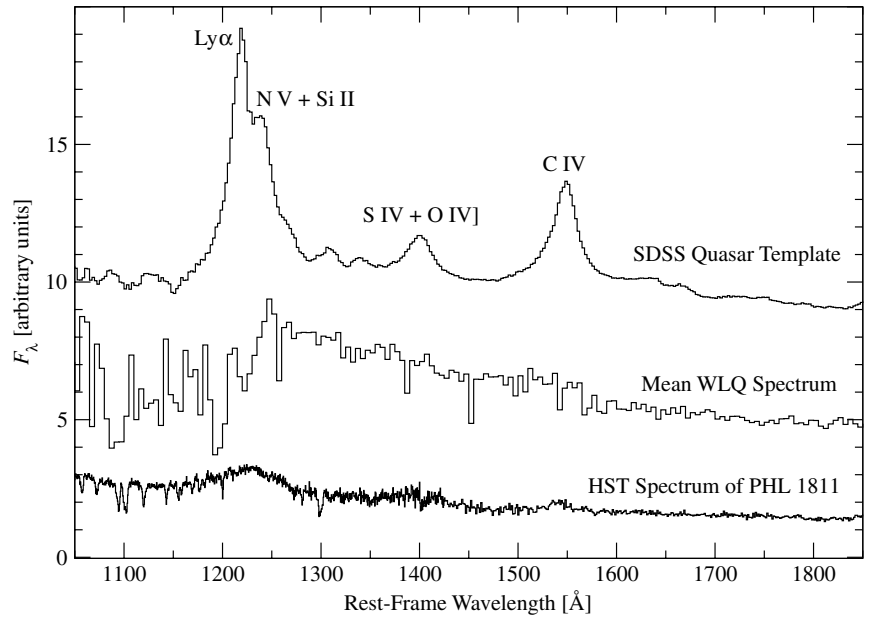

Figure 7. Comparison between the mean UV spectrum of $\sim 2000$ SDSS quasars (top; Vanden Berk et al. 2001), the mean UV spectrum of ten of our WLQs with SDSS spectra (middle; see also Figure 1), and the HST/STIS UV spectrum of PHL 1811 (bottom; Leighly et al. 2007b). All spectra are scaled arbitrarily in flux for clarity. Prominent emission lines are marked on the mean SDSS spectrum.

due to the restricted wavelength range over which this slope can be measured in the optical spectra of high-redshift sources (i.e., given a sufficiently broad wavelength range, the UV continuum slope is $\alpha_{\mathrm{UV}}=-0.5$, and it does not evolve with redshift; see, e.g., Schneider et al. 2005). BL Lacs, on the other hand, are considerably redder in this band (with $\alpha_{\mathrm{o}} \approx-1.5$; e.g., Collinge et al. 2005). The lack of red UV spectra in WLQs may also be reflected by the upper limit we place on the mean column density of neutral absorption in these sources (Section 3.3; Table 4). This is broadly consistent with the idea that the weakness of the emission lines is not a consequence of dust absorption, even though the upper limit on $N_{\mathrm{H}}$ does not provide a tight constraint on allowed reddening in the rest-frame UV.

Another indication that WLQs are related to typical quasars lies in their $\alpha_{\mathrm{ox}}$ distribution (with a spread of $\sim 0.6$ for the radio-quiet sources; see Figure 5). We tested whether this $\alpha_{\mathrm{ox}}$ distribution is consistent with the optically selected sample of Gibson et al. (2008), that includes SDSS quasars with Chandra detections. We performed a KS test and found that the $\alpha_{\mathrm{ox}}$ distributions were consistent between 139 radio-quiet, non-BAL quasars from sample B of Gibson et al. (2008) and our seven radio-quiet WLQs. We found that the null hypothesis, that the two samples were drawn from the same parent distribution, cannot be rejected with a confidence level of $97.3 \%$. After scaling out the dependence of $\alpha_{\text {ox }}$ on optical luminosity (Section 3 ), we also performed a KS test on the two $\Delta \alpha_{\text {ox }}$ distributions of these samples and found that the null hypothesis cannot be rejected with a confidence level of $94.8 \%$.

As noted in S06, at least two sources at low redshift, PG $1407+265$ at $z=0.94$ (McDowell et al. 1995) and PHL 1811 at $z=0.19$ (Leighly et al. 2007b), lack high-ionization emission lines in their UV spectra (although some low-ionization lines are detected in both sources). An additional source, 2QZ J215454.3-305654 at $z=0.494$ (Londish et al. 2004), may also be related to these two, as it shows only an [O III] $\lambda 5007$ emission line; however, since UV spectroscopy has not yet been performed, it is unknown whether it displays higher ionization lines. These three sources, which also appear different from BL Lacs, may perhaps be the low-redshift analogs of the $z \gtrsim 2.2$ WLQs; they are plotted separately in Figure 5. The fact that all 
our WLQs lie at high redshifts is clearly a selection effect due to the requirement of a Lyman break detection. We therefore expect that WLQs should exist also at lower redshifts.

Figure 7 shows a comparison between the UV spectrum of one of these low-redshift WLQs, PHL 1811, and the mean UV spectrum of ten of our high-redshift WLQs (i.e., excluding the Keck spectrum of SDSS J1335+3533) alongside the mean UV spectrum of 'typical' SDSS quasars. Overall, the mean spectrum of our high-redshift WLQs appears similar to the spectrum of PHL 1811, and the high-ionization emission lines in both spectra are significantly weaker compared to the mean SDSS spectrum. The Ly $\alpha+\mathrm{N}$ v complex in PHL 1811 has $\mathrm{EW}=24$ $\AA$, which is somewhat larger than our definition of WLQs (EW $<10 \AA$ ), but the $\mathrm{C}$ IV emission line in that source is exceptionally weak with $E W=6.6 \AA$ (Leighly et al. 2007b). A comprehensive comparison between our high-redshift WLQs and their putative low-redshift counterparts requires rest-frame optical spectroscopy of our sources to search for low-ionization emission lines.

Weak emission lines in AGNs are typically associated with high luminosities in what has been termed the 'Baldwin effect', i.e., the anticorrelation between emission-line EW and quasar luminosity (where the anticorrelation is stronger and steeper for emission lines with higher ionization potentials; e.g., Baldwin 1977; Dietrich et al. 2002). In this context, WLQs might have been expected to dominate the high-luminosity end of the quasar luminosity function, but this is certainly not the case (e.g., Just et al. 2007 studied the properties of the 32 most luminous quasars in SDSS DR3; this sample does not include a single WLQ).

A different interpretation of the Baldwin effect has been suggested by Baskin \& Laor (2004), who have shown that EW(C IV) depends more strongly on the normalized accretion rate (i.e., $L / L_{\text {Edd }}$, where $L$ is the AGN bolometric luminosity) than on $L_{\mathrm{UV}}$ in a sample of 81 low-moderate luminosity AGNs (i.e., with $L \sim 10^{43}-10^{46} \mathrm{erg} \mathrm{s}^{-1}$ ) from Boroson \& Green (1992). Using virial black-hole masses measured from the SDSS spectra of $\sim 60,000$ quasars, Shen et al. (2008) have shown that $L / L_{\text {Edd }}$ exhibits a lognormal distribution (clearly, WLQs could not have been included in their analysis due to their lack of emission lines). Can this be related to the result of DiamondStanic et al. (2009) who found that $\mathrm{EW}(\mathrm{Ly} \alpha+\mathrm{N}$ v) values in SDSS quasars also exhibit a lognormal distribution (Section 1)? In this scenario, WLQs may constitute a significant deviation from the $L / L_{\text {Edd }}$ distribution at the high $-L / L_{\text {Edd }}$ end, in analogy with their significant deviation from the EW distribution at the low-EW end. Testing this idea and the hypothesis that emissionline EW is correlated primarily with $L / L_{\text {Edd }}$ across the entire AGN luminosity range requires extending the Baskin \& Laor (2004) analysis to much higher luminosities, such as those of our WLQs; this is beyond the scope of this work. Near-IR spectroscopy of the $\mathrm{Mg}$ II and $\mathrm{H} \beta$ spectral regions in WLQs is required to determine $L / L_{\text {Edd }}$ values in these sources directly, provided these lines are sufficiently strong to measure (e.g., Shemmer et al. 2004). A high accretion rate has also been proposed for at least one of the low-redshift WLQs, PHL 1811, to explain the absence of the high-ionization emission lines (Leighly et al. 2007b).

If WLQs are AGNs that constitute the high-end tail of the $L / L_{\text {Edd }}$ distribution, then they may also be expected to exhibit steep hard-X-ray continua (see, e.g., Brandt et al. 1997; Shemmer et al. 2008). For example, high-quality XMM-Newton spectra of two of the low-redshift WLQs, PG 1407+265 and PHL 1811, indeed show relatively steep X-ray continua at rest- frame energies $\gtrsim 2 \mathrm{keV}$, with photon indices of $\Gamma=2.19 \pm 0.07$ (Piconcelli et al. 2005) and $\Gamma=2.28_{-0.11}^{+0.12}$ (Leighly et al. 2007a), respectively. High-quality X-ray spectra of high-redshift WLQs have not yet been obtained to test whether they show similar $\mathrm{X}$-ray spectral properties. The X-ray brightest source in our sample, SDSS J1231+0138, had only 110 counts, but it does not exhibit an unusually steep hard-X-ray power-law spectrum $\left(\Gamma=1.76_{-0.34}^{+0.35}\right.$; Section 3.2). However, this WLQ is also the radio-brightest source in our sample (involving perhaps jetrelated emission contributing to its X-ray spectrum). We had already pointed out that our joint spectral fitting of a sample of WLQs does not provide any evidence or hint of a steep hard-X-ray spectrum (Section 3.3; Table 4). Moreover, if we consider WLQs to have $L / L_{\text {Edd }} \gtrsim 1$ (i.e., at the extreme high end of the Shen et al. $2008 L / L_{\text {Edd }}$ distribution), then following the $\Gamma-L / L_{\text {Edd }}$ correlation of Shemmer et al. (2008) these sources should have hard-X-ray slopes with $\Gamma \gtrsim 2.5$. Such steep slopes are rejected with $>99.9 \%$ confidence from our joint spectral fits. Interestingly, however, Table 2 may hint that at least two WLQs, SDSS J0317-0758 and SDSS J0928+1848 (with insufficient counts for a proper spectral analysis) may have steep hard X-ray spectra, based on the effective photon indices obtained from their band ratios. Future XMM-Newton spectroscopic observations of these as well as a statistically representative sample of WLQs are required for a robust test of this idea. Such observations may also provide better constraints on putative Compton reflections in these sources.

\section{SUMMARY}

We present new Chandra observations of nine high-redshift quasars with weak or undetectable high-ionization emission lines in their UV spectra (WLQs). Adding archival X-ray observations of six additional sources of this class has enabled us to place the strongest constraints yet on the X-ray properties of 15 sources of this rare class of AGNs. All but two of these 15 sources are X-ray detected, and for the strongest X-ray (and radio) source we have determined a power-law photon index of $\Gamma=1.76_{-0.34}^{+0.35}$ in the rest-frame $\sim 2-35 \mathrm{keV}$ spectral band. A joint spectral fit of the X-ray spectrum of 11 of these sources gives an upper limit on the mean intrinsic neutral absorption column density of $N_{\mathrm{H}} \lesssim 5 \times 10^{22} \mathrm{~cm}^{-2}$; the corresponding mean power-law photon index is $\Gamma=1.79_{-0.16}^{+0.17}$. These results provide further support to the idea that the weakness of the UV lines is not a consequence of dust absorption, and they show that the mean hard-X-ray spectral slope of WLQs is consistent with the spectral slopes observed in typical RQQs.

Our new X-ray information on high-redshift WLQs has brought us one step closer to distinguishing between two competing models for their nature. Although a boosted-continuum interpretation for the missing emission lines cannot be ruled out based on our current data, we find that WLQs occupy an almost distinct region of the X-ray-radio parameter space from BL Lacs. WLQs are mostly X-ray and radio weak compared to lower-redshift BL Lacs. The strongest argument against the association of WLQs with BL Lacs is the lack of a parent population of X-ray- and radio-bright sources at high redshift. We discuss additional observations that are required to test whether WLQs are quasars with extreme properties. A key goal is to check whether WLQs are quasars with extremely high accretion rates; our current constraints on the mean $\Gamma$ for these sources do not support this idea. Such efforts should provide a better understanding not only of WLQs and the BL Lac phenomena, but of the AGN population as a whole. 
We gratefully acknowledge the financial support of Chandra X-ray Center grant G07-8101X, NASA LTSA grant NAG5-13035 (O.S., W.N.B., D.P.S.), and NSF grant AST0702766 (M.A.S.). We thank Annalisa Celotti, Abe Falcone, and Eric Perlman for fruitful discussions. We also thank an anonymous referee for a thoughtful report that assisted in improving the presentation of this work. Funding for the SDSS and SDSS-II has been provided by the Alfred P. Sloan Foundation, the Participating Institutions, the National Science Foundation, the U.S. Department of Energy, the National Aeronautics and Space Administration, the Japanese Monbukagakusho, the Max Planck Society, and the Higher Education Funding Council for England. The SDSS Web site is http://www.sdss.org/. The SDSS is managed by the Astrophysical Research Consortium for the Participating Institutions. The Participating Institutions are the American Museum of Natural History, Astrophysical Institute Potsdam, University of Basel, University of Cambridge, Case Western Reserve University, University of Chicago, Drexel University, Fermilab, the Institute for Advanced Study, the Japan Participation Group, Johns Hopkins University, the Joint Institute for Nuclear Astrophysics, the Kavli Institute for Particle Astrophysics and Cosmology, the Korean Scientist Group, the Chinese Academy of Sciences (LAMOST), Los Alamos National Laboratory, the Max-Planck-Institute for Astronomy (MPIA), the Max-Planck-Institute for Astrophysics (MPA), New Mexico State University, Ohio State University, University of Pittsburgh, University of Portsmouth, Princeton University, the United States Naval Observatory, and the University of Washington. This research has made use of the NASA/IPAC Extragalactic Database (NED) which is operated by the Jet Propulsion Laboratory, California Institute of Technology, under contract with the National Aeronautics and Space Administration.

\section{REFERENCES}

Anderson, S. F., et al. 2001, AJ, 122, 503

Anderson, S. F., et al. 2007, AJ, 133, 313

Arnaud, K. A. 1996, in ASP Conf. Ser. 101, Astronomical Data Analysis Software and Systems V, ed. G. H. Jacoby \& J. Barnes (San Francisco, CA: ASP), 17

Avni, Y. 1976, ApJ, 210, 642

Baldwin, J. A. 1977, ApJ, 214, 679

Baskin, A., \& Laor, A. 2004, MNRAS, 350, L31

Becker, R. H., White, R. L., \& Helfand, D. J. 1995, ApJ, 450, 559

Bianchi, S., Guainazzi, M., Matt, G., \& Fonseca Bonilla, N. 2007, A\&A, 467, L19

Boroson, T. A., \& Green, R. F. 1992, ApJS, 80, 109

Brandt, W. N., Mathur, S., \& Elvis, M. 1997, MNRAS, 285, L25

Cash, W. 1979, ApJ, 228, 939

Collinge, M. J., et al. 2005, AJ, 129, 2542

Condon, J. J., Cotton, W. D., Greisen, E. W., Yin, Q. F., Perley, R. A., Taylor, G. B., \& Broderick, J. J. 1998, AJ, 115, 1693

Diamond-Stanic, A., et al. 2009, ApJ, submitted

Dickey, J. M., \& Lockman, F. J. 1990, ARA\&A, 28, 215

Dietrich, M., Hamann, F., Shields, J. C., Constantin, A., Vestergaard, M., Chaffee, F., Foltz, C. B., \& Junkkarinen, V. T. 2002, ApJ, 581, 912

Eadie, W. T., Dryard, D., James, F. E., Roos, M., \& Sadoulet, B. 1971, Statistical Methods in Experimental Physics (Amsterdam: North-Holland)

Fan, X., et al. 1999, ApJ, 526, L57

Fan, X., et al. 2001, AJ, 121, 31

Fan, X., et al. 2006, AJ, 131, 1203

Freeman, P. E., Kashyap, V., Rosner, R., \& Lamb, D. Q. 2002, ApJS, 138, 185
Garmire, G. P., Bautz, M. W., Ford, P. G., Nousek, J. A., \& Ricker, G. R 2003, Proc. SPIE, 4851, 28

Gehrels, N. 1986, ApJ, 303, 336

Gibson, R. R., Brandt, W. N., \& Schneider, D. P. 2008, ApJ, 685, 773

Iwasawa, K., \& Taniguchi, Y. 1993, ApJ, 413, L15

Jansen, F., et al. 2001, A\&A, 365, L1

Jiang, L., Fan, X., Ivezić, Ž., Richards, G. T., Schneider, D. P., Strauss, M. A., \& Kelly, B. C. 2007, ApJ, 656, 680

Just, D. W., Brandt, W. N., Shemmer, O., Steffen, A. T., Schneider, D. P., Chartas, G., \& Garmire, G. P. 2007, ApJ, 665, 1004

Kellermann, K. I., Sramek, R., Schmidt, M., Shaffer, D. B., \& Green, R. 1989, AJ, 98, 1195

Kraft, R. P., Burrows, D. N., \& Nousek, J. A. 1991, ApJ, 374, 344

Landt, H., Padovani, P., Perlman, E. S., Giommi, P., Bignall, H., \& Tzioumis, A. 2001, MNRAS, 323, 757

Laurent-Muehleisen, S. A., Kollgaard, R. I., Feigelson, E. D., Brinkmann, W., \& Siebert, J. 1999, ApJ, 525, 127

Leighly, K. M., Halpern, J. P., Jenkins, E. B., Grupe, D., Choi, J., \& Prescott, K. B. 2007a, ApJ, 663, 103

Leighly, K. M., Halpern, J. P., Jenkins, E. B., \& Casebeer, D. 2007b, ApJS, 173, 1

Londish, D., Heidt, J., Boyle, B. J., Croom, S. M., \& Kedziora-Chudczer, L. 2004, MNRAS, 352, 903

Lyons, L. 1991, Data Analysis for Physical Science Students, (Cambridge: Cambridge Univ. Press)

McDowell, J. C., Canizares, C., Elvis, M., Lawrence, A., Markoff, S., Mathur, S., \& Wilkes, B. J. 1995, ApJ, 450, 585

Oke, J. B., \& Gunn, J. E. 1983, ApJ, 266, 713

Padovani, P., Giommi, P., Landt, H., \& Perlman, E. S. 2007, ApJ, 662, 182

Page, K. L., Reeves, J. N., O’Brien, P. T., \& Turner, M. J. L. 2005, MNRAS, 364, 195

Perlman, E. S., Padovani, P., Giommi, P., Sambruna, R., Jones, L. R., Tzioumis, A., \& Reynolds, J. 1998, AJ, 115, 1253

Perlman, E. S., Padovani, P., Landt, H., Stocke, J. T., Costamante, L., Rector, T., Giommi, P., \& Schachter, J. F. 2001, in ASP Conf. Ser. 227, Blazar Demographics and Physics, ed. P. Padovani \& C. M. Urry (San Francisco, CA: ASP) 200

Perlman, E. S., et al. 1996, ApJS, 104, 251

Piconcelli, E., et al. 2005, A\&A, 432, 15

Pier, J. R., Munn, J. A., Hindsley, R. B., Hennessy, G. S., Kent, S. M., Lupton, R. H., \& Ivezić, Ž. 2003, AJ, 125, 1559

Plotkin, R. M., Anderson, S. F., Hall, P. B., Margon, B., Voges, W., Schneider, D. P., Stinson, G., \& York, D. G. 2008, AJ, 135, 2453

Rector, T. A., Stocke, J. T., Perlman, E. S., Morris, S. L., \& Gioia, I. M. 2000, AJ, 120,1626

Reeves, J. N., \& Turner, M. J. L. 2000, MNRAS, 316, 234

Richards, G. T., et al. 2002, AJ, 123, 2945

Sambruna, R. M., Maraschi, L., \& Urry, C. M. 1996, ApJ, 463, 444

Schlegel, D. J., Finkbeiner, D. P., \& Davis, M. 1998, ApJ, 500, 525

Schneider, D. P., et al. 2001, AJ, 121, 1232

Schneider, D. P., et al. 2003, AJ, 126, 2579

Schneider, D. P., et al. 2005, AJ, 130, 367

Schneider, D. P., et al. 2007, AJ, 134, 102

Shemmer, O., Brandt, W. N., Netzer, H., Maiolino, R., \& Kaspi, S. 2008, ApJ, 682,81

Shemmer, O., Netzer, H., Maiolino, R., Oliva, E., Croom, S., Corbett, E., \& di Fabrizio, L. 2004, ApJ, 614, 547

Shemmer, O., et al. 2006, ApJ, 644, 86, (S06)

Shen, Y., Greene, J. E., Strauss, M. A., Richards, G. T., \& Schneider, D. P. 2008, ApJ, 680, 169

Steffen, A. T., Strateva, I., Brandt, W. N., Alexander, D. M., Koekemoer, A. M., Lehmer, B. D., Schneider, D. P., \& Vignali, C. 2006, AJ, 131, 2826

Stickel, M., Fried, J. W., Kuehr, H., Padovani, P., \& Urry, C. M. 1991, ApJ, 374 431

Stocke, J. T., \& Perrenod, S. C. 1981, ApJ, 245, 375

Stocke, J. T. 2001, Blazar Demogr. Phys., 227, 184

Urry, C. M., \& Padovani, P. 1995, PASP, 107, 803

Vanden Berk, D. E., et al. 2001, AJ, 122, 549

Vignali, C., Brandt, W. N., Schneider, D. P., \& Kaspi, S. 2005, AJ, 129, 2519

York, D. G., et al. 2000, AJ, 120, 1579

Zhou, X.-L., \& Wang, J.-M. 2005, ApJ, 618, L83 